\title{
Gas leak due to faulty actuating spindles in a Tec 7 vaporizer
}

\author{
Sumio Amagasa $\cdot$ Eisuke Takahashi $\cdot$ \\ Noriyuki Murata
}

Received: 16 January 2014/ Accepted: 20 March 2014/Published online: 4 April 2014

(C) Japanese Society of Anesthesiologists 2014

Keywords Gas leak - Port valve actuating spindle $\cdot$ Tec 7 vaporizer

To the Editor:

We recently experienced an unexpected gas leak from a vaporizer unit. No significant gas leak from any part of the breathing circuit was detected by the anesthesiologist during his routine pre-use check. However, following induction of anesthesia with propofol and sevoflurane, he noticed that the reservoir bag was gradually deflating, resulting in insufficient ventilation via a face mask despite full closure of the adjustable pressure limiting valve. Initially assuming that the cause of the deflated bag was an inadequate fit of the face mask, the anesthesiologist immediately performed tracheal intubation in the patient and started mechanical ventilation. A few minutes later he noticed that the bellows inside the ventilator was gradually shrinking while the ventilator was operating, implying a significant leak from the gas supply line. The anesthesia machine was replaced as soon as possible.

Electronic supplementary material The online version of this article (doi:10.1007/s00540-014-1825-7) contains supplementary material, which is available to authorized users.

S. Amagasa $(\bowtie) \cdot$ E. Takahashi

Department of Anesthesia, Saitama Prefecture Saiseikai

Kurihashi Hospital, 714-6 Ko-uemon, Kuki, Saitama 349-1105,

Japan

e-mail: amagasa-anes@umin.ac.jp

N. Murata

Faculty of Biomedical Equipment Technology, Saitama

Prefecture Saiseikai Kurihashi Hospital, 714-6 Ko-uemon, Kuki,

Saitama 349-1105, Japan
GE Healthcare Japan, the importer of this anesthesia machine, reported that the major cause of the gas leak was a malfunction of the port valve actuating spindles in a Tec 7 vaporizer. When the vaporizer functions normally, these spindles descend synchronously and equidistantly and open the manifold port valves of the anesthesia machine, thereby enabling fresh gas to flow into the vaporizer once the the vaporizer control dial has been turned on. For some reason that remains unknown, in the present case two spindles, which had been adjusted 9 years previously, descended asynchronously and to different lengths (Electronic Supplementary Material Figs. 1, 2), leading to incomplete sealing around the holes by the flanges of the spindles and, consequently, the escape of gas from the vaporizer. We should be aware that the port valve actuating spindle is an important but potentially hazardous component of a Tec 7 vaporizer.

Conflict of interest None. 